\title{
N92-13607
}

\section{PRODUCTION OF ORGANIC COMPOUNDS IN PLASMAS: A COMPARISON AMONG ELECTRIC SPARKS, LASER-INDUCED PLASMAS AND UV LIGHT}

\author{
T. W. Scattergood" \\ S. U. N. Y. at Stony Brook, \\ C.P. McKay, W. J. Borucki, L.P. Giver, H. Van Ghyseghem \\ NASA Ames Research Center, \\ J.E. Parris \\ SETI Institute \\ and \\ S.L. Miller \\ U. C. San Diego
}

The chemistry in planetary atmospheres that is induced by processes associated with hightemperature plasmas is of broad interest because such processes may explain many of the chemical species observed. There are at least two important phenomena that are known to generate plasmas (and shocks) in planetary atmospheres: lightning and meteor impacts. Lightning has long been recognized to be an important driver of chemical reactions in the Earth's atmosphere and, probably, in the atmospheres of Jupiter, Saturn and Venus. Lightning may also have provided a source for prebiotic compounds on the early Earth. Meteors and cometary objects impacting planetary atmospheres may have supplied prebiotic compounds to or initiated chemical processes in the atmospheres. For both lightning and impacts, rapid heating of atmospheric gases leads to the formation of a high-temperature plasma which emits radiation and produces shock waves that propagate through the surrounding atmosphere. These processes initiate chemical reactions that can transform simple gases into more complex compounds. For example, electric discharges in mixtures of $\mathrm{H}_{2} / \mathrm{CH}_{4} / \mathrm{NH}_{3}$ and $\mathrm{CH}_{4} / \mathrm{N}_{2}$, representing the atmospheres of Jupiter and Titan, respectively, are known to produce a suite of gaseous and condensed phase hydrocarbons and C-N containing compounds. Also, processing of Titan's early atmosphere from impacts of meteors and comets may have been responsible for the molecular nitrogen still there today.

In order to study the production of organic compounds in plasmas (and shocks), various mixtures of $\mathrm{N}_{2}, \mathrm{CH}_{4}$, and $\mathrm{H}_{2}$, modeling the atmosphere of Titan, were exposed to discrete sparks, laser-induced plasmas (LIP) and ultraviolet light. The yields of HCN and simple hydrocarbons were measured and compared to those calculated from a simple quenched thermodynamic equilibrium model. The agreement between experiment and theory was fair for $\mathrm{HCN}$ and $\mathrm{C}_{2} \mathrm{H}_{2}$. However, the yields of $\mathrm{C}_{2} \mathrm{H}_{6}$ and other hydrocarbons were much higher than those predicted by the model. Our experiments suggest that photolysis by ultraviolet light from the plasma is an important process in the synthesis. This was confirmed by the photolysis of gas samples exposed to the light, but not to the plasma or shock waves. The results of these experiments demonstrate that, in addition to the well-known efficient synthesis of organic compounds in plasmas, the yields of saturated species, e.g. ethane, may be higher than predicted by theory and that LIP provide a convenient and clean way of simulating planetary lightning and impact plasmas in the laboratory. 